\title{
Update: Cytokine Dysregulation in Chronic Nonbacterial Osteomyelitis (CNO)
}

\author{
Sigrun R. Hofmann, ${ }^{1}$ Angela Roesen-Wolff, ${ }^{1}$ Gabriele Hahn, ${ }^{2}$ and Christian M. Hedrich ${ }^{1,3}$ \\ ${ }^{1}$ Department of Pediatrics, University Hospital Carl Gustav Carus, Fetscherstraße 74, 01307 Dresden, Germany \\ ${ }^{2}$ Department of Pediatric Radiology, University Hospital Carl Gustav Carus, 01307 Dresden, Germany \\ ${ }^{3}$ Division of Rheumatology, Department of Medicine, Beth Israel Deaconess Medical Center, Harvard Medical School, Boston, \\ MA 02215, USA
}

Correspondence should be addressed to Sigrun R. Hofmann, sigrun.hofmann@uniklinikum-dresden.de

Received 14 January 2012; Accepted 22 March 2012

Academic Editor: Lorenzo Beretta

Copyright ( $) 2012$ Sigrun R. Hofmann et al. This is an open access article distributed under the Creative Commons Attribution License, which permits unrestricted use, distribution, and reproduction in any medium, provided the original work is properly cited.

\begin{abstract}
Chronic nonbacterial osteomyelitis (CNO) with its most severe form chronic recurrent multifocal osteomyelitis (CRMO) is a non-bacterial osteitis of yet unknown origin. Secondary to the absence of both high-titer autoantibodies and autoreactive $\mathrm{T}$ lymphocytes, and the association with other autoimmune diseases, it was recently reclassified as an autoinflammatory disorder of the musculoskeletal system. Since its etiology is largely unknown, the diagnosis is based on clinical criteria, and treatment is empiric and not always successful. In this paper, we summarize recent advances in the understanding of possible etiopathogenetic mechanisms in CNO.
\end{abstract}

\section{Introduction}

Chronic nonbacterial osteomyelitis (CNO) (OMIM number 259680 ) is an autoinflammatory, noninfectious disorder that affects the skeletal system and has first been described in 1972 [1]. An association with further autoimmune and autoinflammatory disorders such as pustolusis palmoplantaris [2], chronic inflammatory bowel disease (IBD), psoriasis, cANCA positive vasculitis, Takayasu's arteriitis, and deficiency of IL-1 receptor antagonist (DIRA) has been discussed [3-5]. CNO covers a wide clinical spectrum from non- or oligosymptomatic monofocal bony lesions to its most severe form chronic recurring multifocal osteomyelitis (CRMO). Generally, any skeletal site can be affected. However, metaphyses of long bones are predominantly involved [6] with a predilection for the lower extremities (distal femur, proximal tibia, distal tibia, and distal fibula [7]), followed by the clavicle and spine. A symmetric involvement is common. Systemic symptoms occur in a subset of patients and include low-grade fevers and malaise. Even though Scully et al. (2008) [7] reported a predominance in females (up to 85\%), larger studies did not confirm this.
However, little is known about the pathophysiology of CNO. Most research articles focus on clinical aspects of CNO, and only few reports discuss putative pathomechanisms underlying this autoinflammatory disorder.

\section{Classification, Clinical Picture, Diagnosis, and Treatment}

Autoinflammatory diseases are rare disorders, characterized by recurrent episodes of fever and inflammation, and mostly affect joints, serosal surfaces, eyes and skin in the absence of high-titer autoantibodies, autoreactive $\mathrm{T}$ lymphocytes [11], and underlying infection [7]. In 2006, McGonagle and McDermott [12] suggested a clinical continuum with autoimmune disorders on the one, and autoinflammatory diseases on the other end of the spectrum. Disorders located towards the middle of the continuum exhibit features of both autoinflammatory as well as autoimmune diseases. According to this definition, $\mathrm{CNO}$ has been classified as an autoinflammatory disorder. In CNO the diagnosis relies on clinical symptoms, systemic inflammation, and elevated 


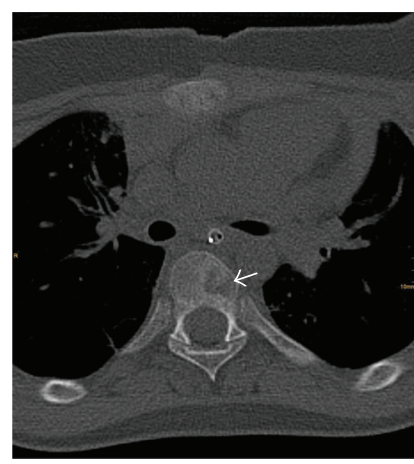

(a)
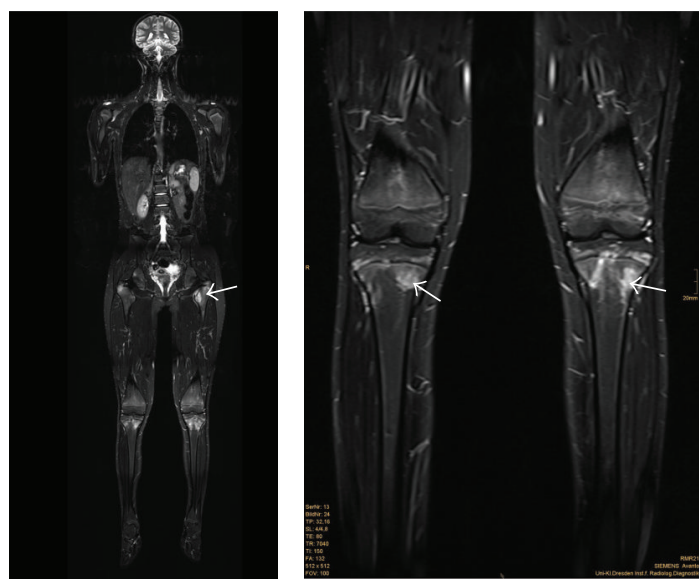

(b)

FIGURE 1: (a) left panel: computerized tomography (CT) of the spine, showing an osteolytic lesions (white arrow) in chronic recurrent multifocal osteomyelitis in a thoracic vertebra. Right panel: radiograph of the spine, showing flattening of the third lumbar vertebra (white arrows) in chronic recurrent multifocal osteomyelitis. (b) magnetic resonance imaging (MRI) in a patient with chronic recurrent multifocal osteomyelitis; left panel: whole-body MRI, showing multiple foci of osteomyelitis, some of which are distributed symmetrically; right panel: magnified image (from the left panel) of the knees, showing inflammatory epiphyseal lesions in both tibiae.

acute phase proteins during attacks. In very few cases, underlying mutations in the relevant genes can be detected (Majeed syndrome, see below). CNO needs to be differentiated from infectious osteomyelitis, including mycobacterial infections, and malignant neoplasms.

Laboratory and histopathological findings are nonspecific, with mildly elevated acute phase markers, and plasmacellular, granulocytic, and monocytic infiltrations respectively [13]. However in a substantial number of patients, systemic inflammatory signs (such as low-grade fever and malaise and elevated acute phase proteins) cannot be found, even during attacks. In these cases, the diagnosis of CNO can be made, combining clinical findings (localized bone pain, swelling, and warmth in the affected areas) and laboratory tests (including testing for mycobacteria) with radiographic techniques. The presence of osteolytic lesions with surrounding sclerosis in X-ray imaging, supplemented by computerized tomography (CT) and/or magnetic resonance imaging (MRI), is usually helpful (Figure 1). Silent asymptomatic lesions frequently appear on nuclear scans.

Even though CNO has been treated with azithromycin [14] in the past, it does generally not respond to antibiotics. Treatment involves anti-inflammatory agents, including NSAIDs $[13,15,16]$ and corticosteroids. In treatment restistant cases, bisphosphonates [17] and biologicals, usually infliximab or etanercept (blocking tumor necrosis factor$\alpha)$ [18] have been applied successfully, suggesting an involvement of TNF- $\alpha$ in the pathophysiology of CNO.

Individual clinical courses of CNO are generally unpredictable. Most cases are self-limiting and eventually resolve without major sequelae. However, a subset of patients exhibit severe and prolonged disease courses and are at risk of developing bone deformities that can be caused by premature closure of the epiphyses, and kyphoscoliosis as a result of vertebral involvement $[4,6,19]$. Disease onset at a young age and high numbers [20] of inflammatory bone lesions at disease onset seem to be predictive of poor outcomes $[4,19]$.

\section{Putative Pathomechanisms}

Imbalances in cytokine expression can cause a disruption of immune homeostasis, resulting in increased or reduced inflammatory responses that in turn may cause susceptibility to infections or autoimmune disorders. Cytokine dysregulation can be caused on various levels, including (1) impaired transcription that can be caused by genomic variation and (2) epigenetic modifications. Genomic variations, including single-nucleotide polymorphisms (SNPs), can influence gene expression by variable transcription factor binding or variation in genome organization [21]. Epigenetic modifications alter chromatin accessibility for transcription factors and/or RNA polymerases [21, 22]. Posttranslational histone modifications influence the transcriptional activity of genes and serve as markers of epigenetic changes. Histone hypermethylation as repressing histone modification mediates dense packing of nucleosomes in transcriptionally silent, socalled "heterochromatic" regions. Activating histone modifications, such as histone acetylation and phosphorylation, mediate "open" or "euchromatic" chromatine conformation that is accessible for transcriptional regulatory factors and RNA polymerases [8].

3.1. Genetic Susceptibility Studies. Genetic studies suggested a predisposing locus for $\mathrm{CNO}$ on chromosome 18q21.3$18 \mathrm{q} 22$ [23]. However, convincing evidence for the involvement of this locus in the pathophysiology of CNO is still lacking. Several observations suggest the contribution of genetic factors to the etiology of CNO.

Ferguson et al. [24, 25] described homozygous mutations in the LPIN2 gene, causing a syndromic autosomal recessive 
form of CNO known as Majeed syndrome. The Majeed syndrome's classical triad includes early-onset CRMO, congenital dyserythropoietic anemia and a neutrophilic dermatosis. It is an extremely rare disease and has only been identified in three unrelated Arabic families $[14,26]$. Bone lesions in Majeed syndrome occur within the first two years of life, and the course is more aggressive then in $\mathrm{CNO}$, with more relapses. LPIN2 was mapped to chromosome $18 \mathrm{p}$ and found to be expressed in almost all tissues [25]. Little is known about the function of LPIN2. It shares a lipin domain with the human ortholog of murine lpin1, which plays a role in fat metabolism. Because of the dyserythropoesis in Majeed syndrome, it has been proposed that LPIN2 could also play a role in mitosis, and that an impaired function may lead to abnormal mitosis in rapidly dividing cells, including the red blood cell lineage in the bone marrow [25]. It has further been hypothesised that mutations in LPIN2 may play an etiologic role in psoriasis, since it localizes to a genomic region identified as a psoriasis susceptibility locus. Furthermore, all carriers of disease causing homozygous LPIN2 mutations exhibit inflammatory dermatoses [25].

Mutations in the pstpip2 gene have been described in the murine form of CNO [27]. To date, there are two murine CNO models, both carrying mutations in the pstpip 2 gene: (1) cmo mice [26-29] and (2) Lupo mice [30]. Cmo (chronic multifocal osteomyelitis) mice have been reported to develop a phenotype that is similar to severe cases of human CNO with multiple sites of inflammation. However, most $\mathrm{CNO}$ patients present with less severe mono- or oligo-focal disease which does not completely resemble the disease in cmo mice that develop multifocal (systemic) inflammatory symptoms. The disease is inherited in an autosomal recessive manner, and the cmo locus has been mapped to the murine chromosome 18 [27]. Within the refined region was the gene pstpip2, which shares significant sequence homology to human PSTPIP1. Expression patterns of murine Pstpip2, also called MAYP (macrophage actin-associated tyrosine-phosphorylated protein), are discussed controversially. While Pstpip2 mRNA is ubiquitously expressed [31], Western blot analysis revealed narrow protein expression patterns, with the highest Pstpip2 expression in myeloid cells [32]. Pstpip2 plays a role in cytoskeletal reorganization, and localizes to the cytosol as an F-actin associated phosphoprotein that interacts with PEST-type protein tyrosine phosphatases (PTP PEST) [31, 32]. The association with PEST family PTPs inhibits tyrosine phosphorylation of wildtype PSTPIP1 [33]. Mutations in the PSTPIP1 gene have been documented to cause the autoinflammatory PAPA syndrome (pyogenic arthritis, pyoderma gangrenosum, and acne). The PSTPIP1 protein binds to pyrin, a regulator that inhibits the NLRP3 inflammasome. Interestingly, Veillette et al. [33] and Wise et al. [34] demonstrated that the PSTPIP1 mutations found in the autoinflammatory PAPA syndrome are in the putative coil-coil domain of PSTPIP1, abolishing the capacity of PSTPIP1 to bind PEST family PTPs. This alteration results in enhanced PSTPIP1 tyrosine phosphorylation and is accompanied by augmented binding of PSTPIP1 to pyrin via an SH3 domain [10], resulting in constitutive activation of NLRP3, production of IL- $1 \beta$, and inflammation [33]
(Figure 2). Some similarities in the phenotypes of cmo mice and (human) PAPA patients may suggest an involvement of the same or similar immunologic pathways. However, the absence of the SH3 domain in Pstpip2 suggests that it does not bind pyrin directly [27]. However, "classical" $\mathrm{CNO}$ patients do not develop the characteristic pustulotic or pyogenic dermatitis that is seen in PAPA patients.

Even though cmo mice serve as an interesting model for bone inflammation, none of the reported CNO patients [35] carry mutation in the PSTPIP1 and PSTPIP2 genes, except for several known polymorphisms.

The recent report of a condition, presenting with neonatal onset sterile multifocal osteomyelitis, periostosis, and pustulosis, that is caused by a deficiency of IL-1 receptor antagonist (DIRA), is of special interest [5]. Because of similar clinical, radiological and histological features of $\mathrm{CNO}$ and DIRA, both disorders might share common or similar autoinflammatory processes. However, in a recent study [36] a heterozygous missense mutation c.281G $>\mathrm{T}$ (p.Cys94Phe) could only be found in one patient with $\mathrm{CNO}$. In the other patients, only frequent polymorphisms were found.

Another hint towards the understanding of genetic contributors to the pathophysiology of $\mathrm{CNO}$ may be the clinical similarities of CNO patients and patients with hypophosphatasia [37, 38]. Hypophosphatasia is a rare defect of bone metabolism that is caused by a number of mutations in the gene encoding for the tissue-nonspecific alkaline phosphatase (TNSALP) on chromosome 1p36.1. The mode of inheritance is mostly autosomal-recessive [37, $39,40]$. TNSALP mutations result in reduced enzyme activity and elevated concentrations of TNSALP substrates, including inorganic pyrophosphate, pyridoxal-50-phosphate and phosphoethanolamine [41-43]. It has been documented that pyrophosphate crystals stimulate the NLRP3 inflammasome resulting in Interleukin-1 production [44]. An inflammatory process secondary to the metabolic defect in hypophosphatasia involving the NLRP3 inflammasome may therefore be involved in the pathogenesis of CNO.

Since associations between CNO and Crohn's disease, a multifactorial autoinflammatory disease, have been reported [26], Morbach and colleagues [45] analyzed CNO patients for NOD2/CARD15 mutations. However, CNO without intestinal inflammation was not associated with common CARD15 gene variants [45].

In addition, susceptibility regions have been identified on chromosome 18 in two other diseases sharing similarities with CNO: familial expansile osteolysis and familial Paget's disease of bone $[26,46,47]$. The phenotypes of both diseases have been linked to mutations in the TNFRSF11A gene at 18q22.1, which encodes the receptor activator of nuclear factor kappa B (RANK) [46, 47].

Recently, we reported an association of CNO with IL10 promoter polymorphisms [3, 8]. IL-10 is an immuneregulatory cytokine that controls inflammation by limiting inflammatory cytokine expression and antigen presentation. Dysregulation in IL-10 expression, sometimes caused by single-nucleotide polymorphisms (SNPs) in the IL10 promoter have been associated to autoimmune and infectious diseases, such as SLE [48], asthma, chronic viral diseases, 


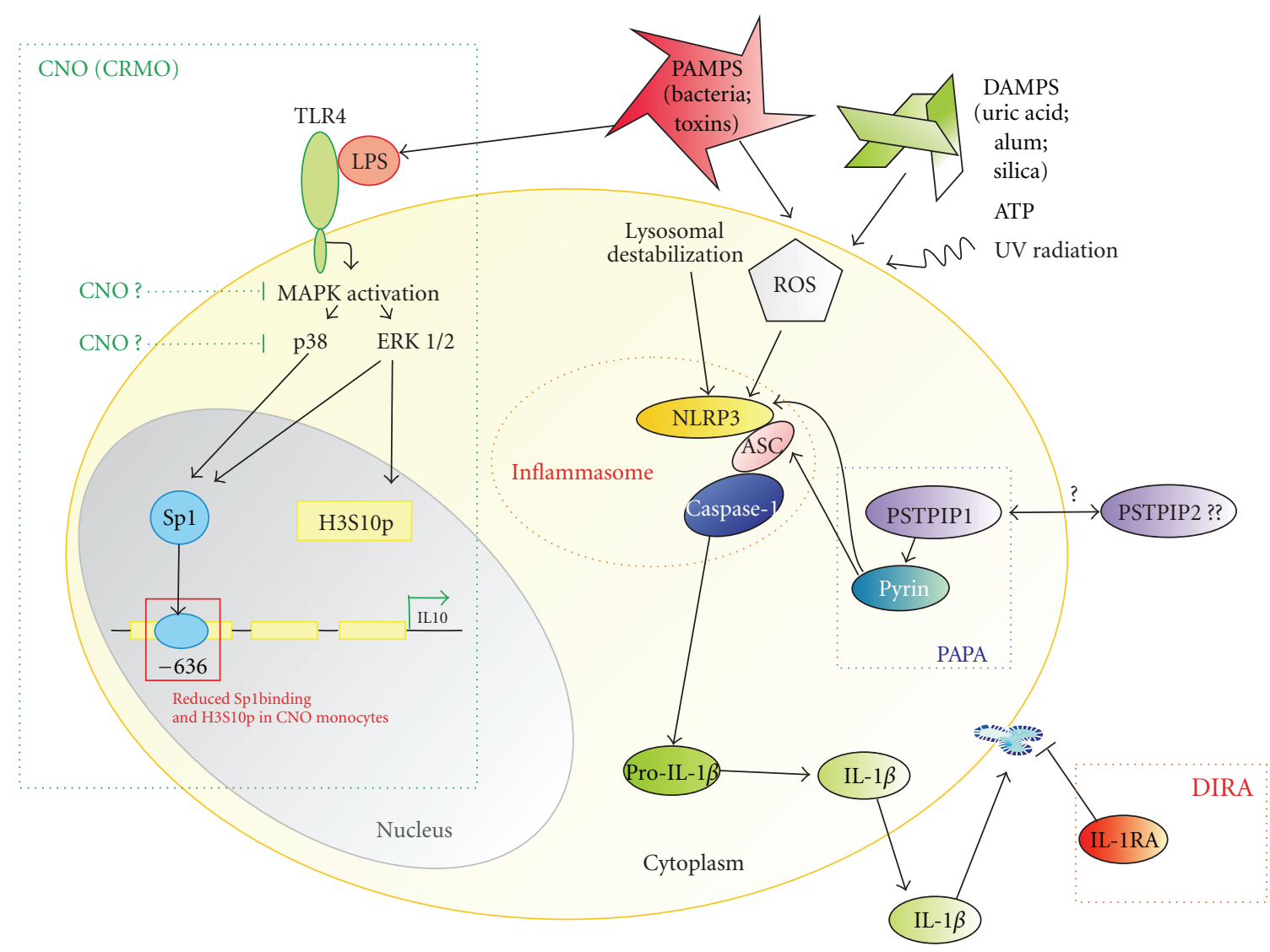

FIGURE 2: Possible pathophysiological mechanisms in autoinflammatory diseases with emphasis on CNO. Modified from [8, 9]. Endogenous and exogenous danger signals (danger-associated molecular patterns: DAMPs and pathogen-associated molecular patterns: PAMPs) activate the NLRP3 inflammasome that is involved in various autoinflammatory disorders. The exact pathways that result in NLRP3 activation are yet unknown. However, reactive oxygen species (ROS) and lysosomal destabilization seem to be involved. The selected monogenic autoinflammatory syndromes result in an activation of the caspase-1 complex. In PAPA, mutations in PSTPIP1 lead to prolonged binding of PSTPIP1 to pyrin via an SH3 domain [10] and impairment of pyrin function, resulting in constitutive activation of NLRP3, production of IL-1 $\beta$, and inflammation. IL-1 receptor antagonist (IL-1RA) is a naturally occurring IL-1 $\beta$ antagonist, and mutations in the IL1RA gen lead to DIRA. Based on the finding that Sp1 binding to the IL10 promoter is reduced, and H3S10 phosphorylation is impaired in the same region in monocytes from $C N O$ patients, we concluded that processes upstream of Sp1 activation and H3S10 phosphorylation may be involved. A reduced activity of MAP kinases upstream of Sp1 signalling may be responsible, resulting in reduced Sp1 activation and reduced H3S10 phosphorylation of the IL10 proximal promoter. ASC: apoptosis-associated speck-like protein containing a caspase recruitment domain; Casp1: enzymatically active caspase-1; IL-18: interleukin-18; IL-1 $\beta$ : interleukin-1 $\beta$; NLRP3: NOD-like receptor family, pyrin domain containing 3; pro-Casp1: procaspase-1; ROS: reactive oxygen species; CNO: chronic nonbacterial osteomyelitis; DIRA: deficiency in IL-1 receptor antagonist; PAPA: pyogenic arthritis, pyoderma gangrenosum and acne; PSTPIP1: proline-serine-threonine phosphataseinteracting protein 1 .

rheumatoid arthritis as well as transplantation complications and cancer [49-51].

IL10 promoter polymorphisms as an example for genomic variation, appear to play an important role in the interindividual variation of IL-10 expression $[8,52]$. The IL10 promoter polymorphisms $-1082 \mathrm{G}>\mathrm{A}$ ( $\mathrm{rs} 1800869)$, $-819 \mathrm{C}>\mathrm{T}$ (rs1800871), and $-592 \mathrm{C}>\mathrm{A}(\mathrm{rs} 1800872)$ are in tight linkage disequilibrium and form three well-defined or "classical" haplotypes: ATA, ACC, and GCC [3]. ATA is associated with low, ACC with intermediate, and GCC with high levels of IL-10 expression $[48,53,54]$. However, for the interindividual variability of IL-10 expression, additional host factors seem to play an important role [52].
As aforementioned, an association of IL10 promoter polymorphisms with $\mathrm{CNO}$ has been described [8], demonstrating an increased frequency of high IL-10 expressing $-1082 \mathrm{G} / \mathrm{G}$ alleles, and resulting GCC haplotypes. Interestingly, monocytes from $\mathrm{CNO}$ patients failed to produce IL-10 in response to stimulation with LPS. This could be explained by (1) IL10 promoter polymorphisms as part of larger haplotypes, extending several hundred kilobases over the entire cytokine cluster, develop cumulative effects in a genomic setting [8]. This hypothesis is supported by a recent study [55] that failed to find a link between IL10 promoter SNPs and inflammatory bowel disease (IBD), but showed an association between a SNP $3^{\prime}$ to the IL10 gene and 
ulcerative colitis. (2) since it is known that IL-10 mediates the proliferation of various lymphocytic tissues, including $\mathrm{B}$ cells, and enhances antibody production [56]. Lymphocytes, but also plasma cells can be predominantly found in biopsies, taken from CNO lesions. Having the anti-inflammatory aspects of IL-10 expression in mind, an association with GCC haplotypes could partly explain the lymphocyte expansion in affected tissues [6]. (3) additional, namely, epigenetic mechanisms, could be involved in the dysregulation of cytokine expression in CNO (see below).

3.2. Epigenetic Mechanisms. The expression of pro- and antiinflammatory cytokines, including IL-10, is considered to be under epigenetic control. Zhang et al. [57] reported histone-phosphorylation within the IL10 proximal promoter to be associated with transcription. Histone phosphorylation is particularly increased in regulatory regions that bind the transcription factor specificity protein 1 (Sp1) and 3 (Sp3) and mediate transcriptional activation in response to stimulation with LPS [58-61]. We recently demonstrated that monocytes from CNO patients fail to produce IL- 10 . Since the proximal promoter region of IL10 displays multiple GC rich sequences, which bind SP-family transcription factors, the binding of the transcriptional regulatory factor Sp1 (previously reported to play a role in the regulation of IL-10 in monocytes and macrophages [55]) to the IL10 promoter has been investigated. A markedly reduced Sp1 recruitment to the IL10_636 Sp1 element has been detected in monocytes from CNO patients in response to LPS stimulation [8]. This was accompanied with reduced histone $\mathrm{H} 3$ serine 10 phosphorylation (H3S10p), an activating epigenetic mark. These findings indicate (1) reduced Sp1 recruitment and (2) attenuated $\mathrm{H} 3 \mathrm{~S} 10$ phosphorylation around the IL10_636 element within the IL10 promoter [8].

Given that (1) LPS stimulation induces MAP kinase (MAPK) activation in monocytes and macrophages, including the p38 [61], the ERKs 1 and 2, and the JNK MAPK pathway [58], and (2) ERKs 1 and 2 are implicated in H3S10 phosphorylation [62], these reports could indicate a new pathophysiological mechanism in CNO with impaired MAPK signaling, resulting in reduced H3S10 phosphorylation, and $\mathrm{Sp} 1$ recruitment to the IL10 promoter and subsequently attenuated gene expression and immune homeostasis [8].

3.3. Other Possible Etiopathogenetic Mechanisms of CNO. TNF- $\alpha$ and IL- $1 \beta$ are implicated in the pathophysiology of various autoimmune and autoinflammatory disorders, including $\mathrm{CNO}$, where it has been documented to be increased in inflammatory lesions [35]. Furthermore, clinical response to anti-TNF $\alpha$ or IL-1 receptor antagonist (IL1ra) anakinra treatment has been documented in $\mathrm{CNO}$ (summarized in [63]). However, it remains unclear whether increased TNF- $\alpha$ and IL- $1 \beta$ expression is the pathophysiological cause of $\mathrm{CNO}$ or a downstream event in the disease. The aforementioned reduced expression of IL-10 in monocytes from $\mathrm{CNO}$ patients may well contribute to a cytokine imbalance towards proinflammatory signals, including TNF- $\alpha$.

\section{Conclusions}

Only few CNO cases have been associated with mutations in single genes. However, they do not explain the pathophysiology of $\mathrm{CNO}$ in the vast majority of cases. Thus, neither the etiology nor the pathogenesis of CNO is completely understood. Mutations in the TNF $\alpha$ and ILIRA genes in humans and the Pstpip2 gene in mice appear to be promising in the search for pathomechanisms in CNO. The most recently described pathophysiological mechanism in CNO might involve impaired MAPK signaling, reduced H3S10 phosphorylation, and attenuated Sp1 recruitment to the IL10 promoter that results in an impaired gene expression.

\section{Conflict of Interests}

The authors declare that they have no financial conflict of interests.

\section{Acknowledgments}

All authors are employees of the University Hospital Carl Gustav Carus, Dresden. The research of S. R. Hofmann and A. Roesen-Wolff is supported by the German Research Foundation (Klinische Forschergruppe KFO 249, project TP2, HO 4510/1-1 and TP1, RO/471-11). The work of Christian M. Hedrich was supported by MeDDrive 2010, University of Technology Dresden, Germany.

\section{References}

[1] A. Giedion, W. Holthusen, L. F. Masel, and D. Vischer, "Subacute and chronic "symmetrical" osteomyelitis," Annales de Radiologie, vol. 15, no. 3, pp. 329-342, 1972.

[2] B. Bjorksten, K. H. Gustavson, B. Eriksson, A. Lindholm, and S. Nordström, "Chronic recurrent multifocal osteomyelitis and pustulosis palmoplantaris," Journal of Pediatrics, vol. 93, no. 2, pp. 227-231, 1978.

[3] J. Hamel, D. Paul, M. Gahr, and C. M. Hedrich, "Pilot study: possible association of IL10 promoter polymorphisms with CRMO," Rheumatology International, vol. 32, no. 2, pp. 555556, 2011.

[4] R. S. Iyer, M. M. Thapa, and F. S. Chew, "Chronic recurrent multifocal osteomyelitis: review," American Journal of Roentgenology, vol. 196, no. 6, supplement, pp. S87-S91, 2011.

[5] I. Aksentijevich, S. L. Masters, P. J. Ferguson et al., "An autoinflammatory disease with deficiency of the interleukin1-receptor antagonist," The New England Journal of Medicine, vol. 360, no. 23, pp. 2426-2437, 2009.

[6] A. G. Jurik, "Chronic recurrent multifocal osteomyelitis," Seminars in Musculoskeletal Radiology, vol. 8, no. 3, pp. 243253, 2004.

[7] C. Scully, T. Hodgson, and H. Lachmann, "Auto-inflammatory syndromes and oral health," Oral Diseases, vol. 14, no. 8, pp. 690-699, 2008. 
[8] S. R. Hofmann, T. Schwarz, J. C. Moller, H. Morbach, A. Schnabel et al., "Chronic non-bacterial osteomyelitis is associated with impaired Sp1 signaling, reduced IL10 promoter phosphorylation, and reduced myeloid IL10 expression," Clinical Immunology, vol. 3, pp. 317-327, 2011.

[9] S. R. Hofmann, M. C. Heymann, A. Hermsdorf, and A. Roesen-Wolff, "Recent advances in autoinflammatory diseases and animal models," Journal of Genetic Syndromes \& Gene Therapy, vol. S3, article 002, 2011.

[10] N. G. Shoham, M. Centola, E. Mansfield et al., "Pyrin binds the PSTPIP1/CD2BP1 protein, defining familial Mediterranean fever and PAPA syndrome as disorders in the same pathway," Proceedings of the National Academy of Sciences of the United States of America, vol. 100, no. 23, pp. 13501-13506, 2003.

[11] D. L. Kastner, I. Aksentijevich, and R. Goldbach-Mansky, "Autoinflammatory disease reloaded: a clinical perspective," Cell, vol. 140, no. 6, pp. 784-790, 2010.

[12] D. McGonagle and M. F. McDermott, "A proposed classification of the immunological diseases," PLoS Medicine, vol. 3, no. 8, article e297, 2006.

[13] H. J. Girschick, C. Zimmer, G. Klaus, K. Darge, A. Dick, and H. Morbach, "Chronic recurrent multifocal osteomyelitis: what is it and how should it be treated?" Nature Clinical Practice Rheumatology, vol. 3, no. 12, pp. 733-738, 2007.

[14] M. Twilt and R. M. Laxer, "Clinical care of children with sterile bone inflammation," Current Opinion in Rheumatology, vol. 23, no. 5, pp. 424-431, 2011.

[15] S. Weihe, H. Eufinger, O. Terhaar, M. Konig, and E. Machtens, "Mandibular involvement in chronic recurrent multifocal osteomyelitis (CRMO) in adulthood," Mund Kiefer Gesichtschir, vol. 4, no. 3, pp. 187-192, 2000.

[16] J. C. Abril and A. Ramirez, "Successful treatment of chronic recurrent multifocal osteomyelitis with indomethacin: a preliminary report of five cases," Journal of Pediatric Orthopaedics, vol. 27, no. 5, pp. 587-591, 2007.

[17] S. Compeyrot-Lacassagne, A. M. Rosenberg, P. Babyn, and R. M. Laxer, "Pamidronate treatment of chronic noninfectious inflammatory lesions of the mandible in children," Journal of Rheumatology, vol. 34, no. 7, pp. 1585-1589, 2007.

[18] A. Deutschmann, C. J. Mache, K. Bodo, D. Zebedin, and E. Ring, "Successful treatment of chronic recurrent multifocal osteomyelitis with tumor necrosis factor- $\alpha$ blockage," Pediatrics, vol. 116, no. 5, pp. 1231-1233, 2005.

[19] R. S. Iyer, M. M. Thapa, and F. S. Chew, "Imaging of chronic recurrent multifocal osteomyelitis: self-assessment module," American Journal of Roentgenology, vol. 196, no. 6, supplement, pp. WS62-WS65, 2011.

[20] C. Catalano-Pons, A. Comte, J. Wipff et al., "Clinical outcome in children with chronic recurrent multifocal osteomyelitis," Rheumatology, vol. 47, no. 9, pp. 1397-1399, 2008.

[21] C. M. Hedrich and J. H. Bream, "Cell type-specific regulation of IL-10 expression in inflammation and disease," Immunologic Research, vol. 47, no. 1-3, pp. 185-206, 2010.

[22] C. Brenner and F. Fuks, "A methylation rendezvous: reader meets writers," Developmental Cell, vol. 12, no. 6, pp. 843-844, 2007.

[23] A. Golla, A. Jansson, J. Ramser et al., "Chronic recurrent multifocal osteomyelitis (CRMO): evidence for a susceptibility gene located on chromosome 18q21.3-18q22," European Journal of Human Genetics, vol. 10, no. 3, pp. 217-221, 2002.

[24] H. I. El-Shanti and P. J. Ferguson, "Chronic recurrent multifocal osteomyelitis: a concise review and genetic update," Clinical Orthopaedics and Related Research, vol. 462, pp. 11-19, 2007.
[25] P. J. Ferguson, S. Chen, M. K. Tayeh et al., "Homozygous mutations in LPIN2 are responsible for the syndrome of chronic recurrent multifocal osteomyelitis and congenital dyserythropoietic anaemia (Majeed syndrome)," Journal of Medical Genetics, vol. 42, no. 7, pp. 551-557, 2005.

[26] J. Wipff, C. Adamsbaum, A. Kahan, and C. Job-Deslandre, "Chronic recurrent multifocal osteomyelitis," Joint Bone Spine, vol. 78, no. 6, pp. 555-560, 2011.

[27] P. J. Ferguson, X. Bing, M. A. Vasef et al., "A missense mutation in pstpip2 is associated with the murine autoinflammatory disorder chronic multifocal osteomyelitis," Bone, vol. 38, no. 1, pp. 41-47, 2006.

[28] L. Byrd, M. Grossmann, M. Potter, and G. L. Shen-Ong, "Chronic multifocal osteomyelitis, a new recessive mutation on chromosome 18 of the mouse," Genomics, vol. 11, no. 4, pp. 794-798, 1991.

[29] T. A. Hentunen, S. J. Choi, B. F. Boyce et al., "A murine model of inflammatory bone disease," Bone, vol. 26, no. 2, pp. 183$188,2000$.

[30] J. Grosse, V. Chitu, A. Marquardt et al., "Mutation of mouse Mayp/Pstpip2 causes a macrophage autoinflammatory disease," Blood, vol. 107, no. 8, pp. 3350-3358, 2006.

[31] Y. Wu, D. Dowbenko, and L. A. Lasky, "PSTPIP 2, a second tyrosine phosphorylated, cytoskeletal-associated protein that binds a PEST-type protein-tyrosine phosphatase," The Journal of Biological Chemistry, vol. 273, no. 46, pp. 30487-30496, 1998.

[32] Y. G. Yeung, S. Soldera, and E. R. Stanley, "A novel macrophage actin-associated protein (MAYP) is tyrosine- phosphorylated following colony stimulating factor-1 stimulation," The Journal of Biological Chemistry, vol. 273, no. 46, pp. 30638-30642, 1998.

[33] A. Veillette, I. Rhee, C. M. Souza, and D. Davidson, "PEST family phosphatases in immunity, autoimmunity, and autoinflammatory disorders," Immunological Reviews, vol. 228, no. 1, pp. 312-324, 2009.

[34] C. A. Wise, J. D. Gillum, C. E. Seidman et al., "Mutations in CD2BP1 disrupt binding to PTP PEST and are responsible for PAPA syndrome, an autoinflammatory disorder," Human Molecular Genetics, vol. 11, no. 8, pp. 961-969, 2002.

[35] A. Jansson, E. D. Renner, J. Ramser et al., "Classification of non-bacterial osteitis: retrospective study of clinical, immunological and genetic aspects in 89 patients," Rheumatology, vol. 46, no. 1, pp. 154-160, 2007.

[36] C. Beck, H. J. Girschick, H. Morbach, T. Schwarz, and T. Yimam, "Mutation screening of the IL-1 receptor antagonist gene in chronic non-bacterial osteomyelitis of childhood and adolescence," Clinical and Experimental Rheumatology, vol. 6, pp. 1040-1043, 2011.

[37] M. P. Whyte, D. Wenkert, W. H. McAlister et al., "Chronic recurrent multifocal osteomyelitis mimicked in childhood hypophosphatasia," Journal of Bone and Mineral Research, vol. 24, no. 8, pp. 1493-1505, 2009.

[38] C. Beck, H. Morbach, P. Richl, M. Stenzel, and H. J. Girschick, "How can calcium pyrophosphate crystals induce inflammation in hypophosphatasia or chronic inflammatory joint diseases?" Rheumatology International, vol. 29, no. 3, pp. 229238, 2009.

[39] C. Beck, H. Morbach, M. Stenzel et al., "Hypophosphatasia," Klinische Padiatrie, vol. 221, no. 4, pp. 219-226, 2009.

[40] H. J. Girschick, E. Mornet, M. Beer, M. Warmuth-Metz, and P. Schneider, "Chronic multifocal non-bacterial osteomyelitis in hypophosphatasia mimicking malignancy," BMC Pediatrics, vol. 7, article 3, 2007. 
[41] E. Mornet, "Hypophosphatasia," Orphanet Journal of Rare Diseases, vol. 40, 2007.

[42] I. Brun-Heath, A. S. Lia-Baldini, S. Maillard et al., "Delayed transport of tissue-nonspecific alkaline phosphatase with missense mutations causing hypophosphatasia," European Journal of Medical Genetics, vol. 50, no. 5, pp. 367-378, 2007.

[43] C. Beck, H. Morbach, C. Wirth, M. Beer, and H. J. Girschick, "Whole-body MRI in the childhood form of hypophosphatasia," Rheumatology International, vol. 10, pp. 1315-1320, 2011.

[44] B. Pazar, H. K. Ea, S. Narayan et al., "Basic calcium phosphate crystals induce monocyte/macrophage IL- $1 \beta$ secretion through the NLRP3 inflammasome in vitro," Journal of Immunology, vol. 186, no. 4, pp. 2495-2502, 2011.

[45] H. Morbach, A. Dick, C. Beck et al., "Association of chronic non-bacterial osteomyelitis with Crohn's disease but not with CARD15 gene variants," Rheumatology International, vol. 30, no. 5, pp. 617-621, 2010.

[46] A. E. Hughes, S. H. Ralston, J. Marken et al., "Mutations in TNFRSF11A, affecting the signal peptide of RANK, cause familial expansile osteolysis," Nature Genetics, vol. 24, no. 1, pp. 45-48, 2000.

[47] A. B. Sparks, S. N. Peterson, C. Bell et al., "Mutation screening of the TNFRSF11A gene encoding receptor activator of NFkB (RANK) in familial and sporadic Paget's disease of bone and osteosarcoma," Calcified Tissue International, vol. 68, no. 3, pp. 151-155, 2001.

[48] A. W. Gibson, J. C. Edberg, J. Wu, R. G. Westendorp, T. W. Huizinga, and R. P. Kimberly, "Novel single nucleotide polymorphisms in the distal IL-10 promoter affect IL-10 production and enhance the risk of systemic lupus erythematosus," Journal of Immunology, vol. 166, no. 6, pp. 3915-3922, 2001.

[49] J. Eskdale, P. Wordsworth, S. Bowman, M. Field, and G. Gallagher, "Association between polymorphisms at the human IL10 locus and systemic lupus erythematosus," Tissue Antigens, vol. 49, no. 6, pp. 635-639, 1997.

[50] R. Lazarus, D. Vercelli, L. J. Palmer et al., "Single nucleotide polymorphisms in innate immunity genes: abundant variation and potential role in complex human disease," Immunological Reviews, vol. 190, pp. 9-25, 2002.

[51] A. P. Vicari and G. Trinchieri, "Interleukin-10 in viral diseases and cancer: exiting the labyrinth?" Immunological Reviews, vol. 202, pp. 223-236, 2004.

[52] E. Reuss, R. Fimmers, A. Kruger, C. Becker, C. Rittner, and T. Höhler, "Differential regulation of interleukin-10 production by genetic and environmental factors-a twin study," Genes and Immunity, vol. 3, no. 7, pp. 407-413, 2002.

[53] M. Mormann, H. Rieth, T. D. Hua et al., "Mosaics of gene variations in the interleukin-10 gene promoter affect interleukin10 production depending on the stimulation used," Genes and Immunity, vol. 5, no. 4, pp. 246-255, 2004.

[54] A. Salhi, V. Rodrigues Jr., F. Santoro et al., "Immunological and genetic evidence for a crucial role of IL10 in cutaneous lesions in humans infected with Leishmania braziliensis," Journal of Immunology, vol. 180, no. 9, pp. 6139-6148, 2008.

[55] A. Franke, T. Balschun, T. H. Karlsen et al., "Sequence variants in IL10, ARPC2 and multiple other loci contribute to ulcerative colitis susceptibility," Nature Genetics, vol. 40, no. 11, pp. 1319-1323, 2008.

[56] C. M. Hedrich, A. Ramakrishnan, D. Dabitao, F. Wang, D. Ranatunga, and J. H. Bream, "Dynamic DNA methylation patterns across the mouse and human IL10 genes during CD4 ${ }^{+}$ T cell activation; influence of IL-27," Molecular Immunology, vol. 48 , no. $1-3$, pp. 73-81, 2010.
[57] X. Zhang, J. P. Edwards, and D. M. Mosser, "Dynamic and transient remodeling of the macrophage IL-10 promoter during transcription," Journal of Immunology, vol. 177, no. 2, pp. 1282-1288, 2006.

[58] H. D. Brightbill, S. E. Plevy, R. L. Modlin, and S. T. Smale, "A prominent role for Sp1 during lipopolysaccharide-mediated induction of the IL-10 promoter in macrophages," Journal of Immunology, vol. 164, no. 4, pp. 1940-1951, 2000.

[59] W. Ma, W. Lim, K. Gee et al., "The p38 mitogen-activated kinase pathway regulates the human interleukin10 promoter via the activation of $\mathrm{Sp} 1$ transcription factor in lipopolysaccharide-stimulated human macrophages," The Journal of Biological Chemistry, vol. 276, no. 17, pp. 1366413674, 2001.

[60] J. W. Steinke, E. Barekzi, J. Hagman, and L. Borish, "Functional analysis of -571 IL-10 promoter polymorphism reveals a repressor element controlled by Sp1," Journal of Immunology, vol. 173, no. 5, pp. 3215-3222, 2004.

[61] H. Chanteux, A. C. Guisset, C. Pilette, and Y. Sibille, "LPS induces IL-10 production by human alveolar macrophages via MAPKinases- and Sp1-dependent mechanisms," Respiratory Research, vol. 8, article 71, 2007.

[62] M. Lucas, X. Zhang, V. Prasanna, and D. M. Mosser, "ERK activation following macrophage Fc $\gamma \mathrm{R}$ ligation leads to chromatin modifications at the IL-10 locus," Journal of Immunology, vol. 175, no. 1, pp. 469-477, 2005.

[63] D. Eleftheriou, T. Gerschman, N. Sebire, P. Woo, C. A. Pilkington, and P. A. Brogan, "Biologic therapy in refractory chronic non-bacterial osteomyelitis of childhood," Rheumatology, vol. 49, no. 8, pp. 1505-1512, 2010. 


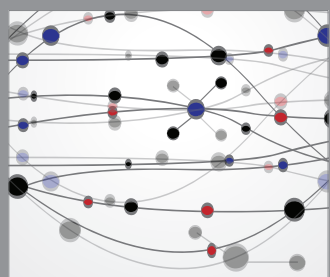

The Scientific World Journal
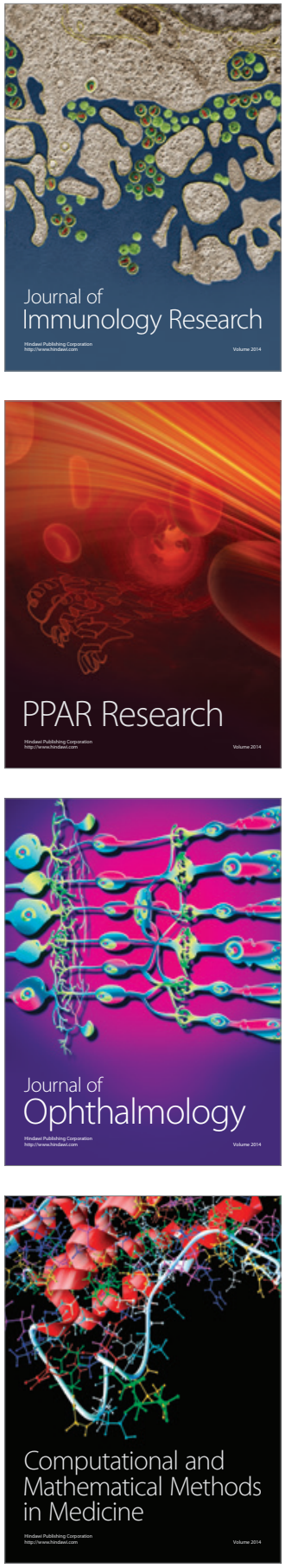

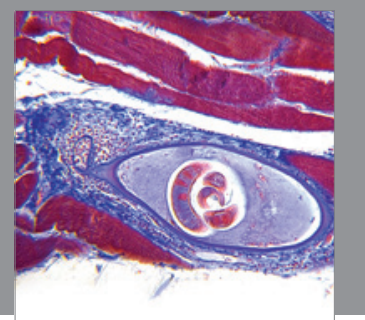

Gastroenterology

Research and Practice
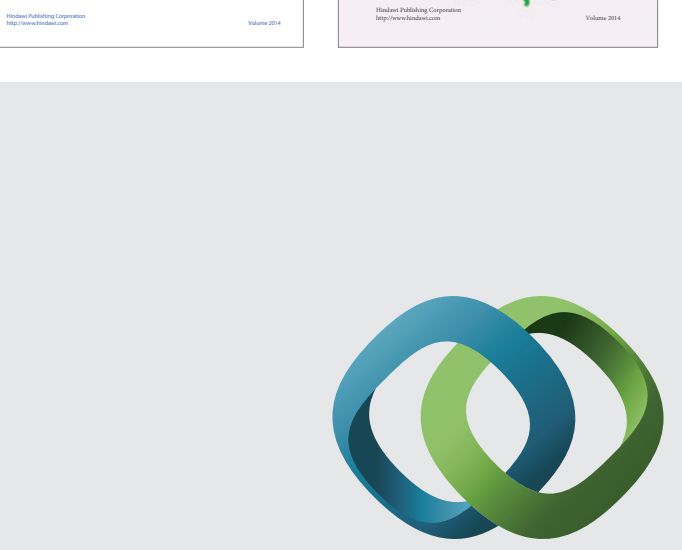

\section{Hindawi}

Submit your manuscripts at

http://www.hindawi.com
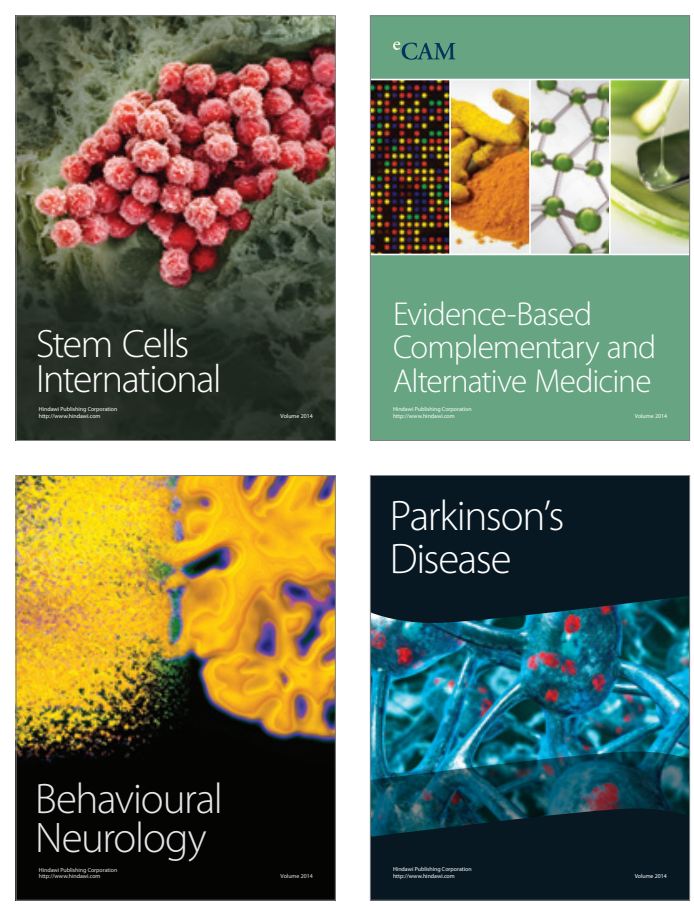

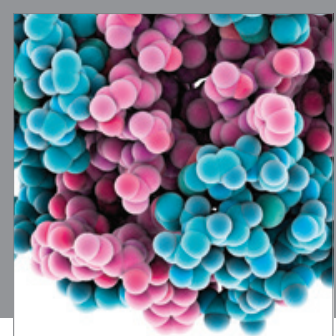

Journal of
Diabetes Research

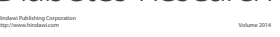

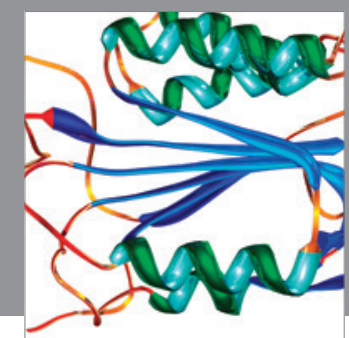

Disease Markers
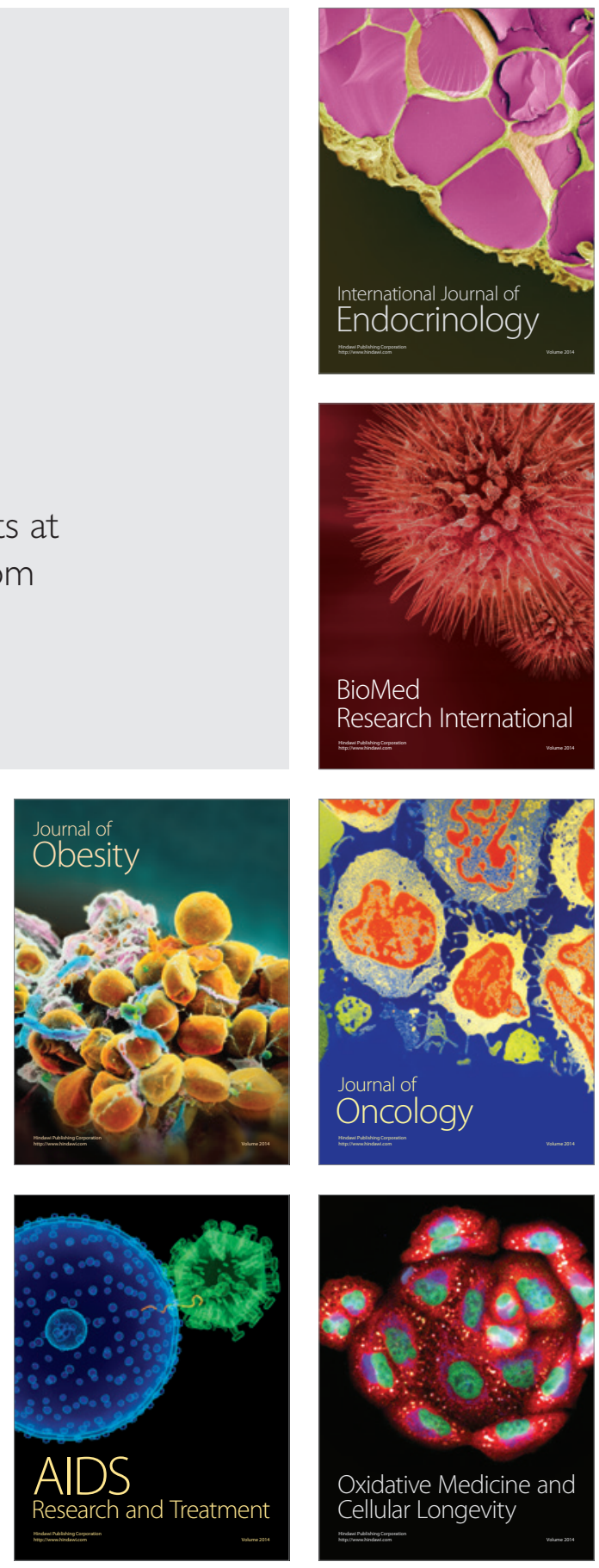